INTERNATIONAL JOURNAL OF RESEARCHES IN BIOSCIENCES, AGRICULTURE AND TECHNOLOGY (C) VISHWASHANTI MULTIPURPOSE SOCIETY (Global Peace Multipurpose Society) R. No. MH-659/13(N) www.vmsindia.org

\title{
GROWTH AND CHARECTRIZATION OF TECHNOLOGICALY IMPORTANT NON-LINEAR OPTICAL CRYSTALS
}

\author{
A. P. Bhat and K. G. Rewatkar \\ Department of Physics, Dr. Ambedkar College, Deekshabhoomi, Nagpur
}

\begin{abstract}
:
Modern days of technologies non linear optical (NLO) crystals plays important role. Application of NLO crystal in optoelectronics, photonics, semiconductor, optics industry . mostly use of NLO crystal in modern LASER technology such as laser spectroscopy, photolithography, semiconductor high frequency laser, laser surgery, internal confinement of fusion reactors and various basic properties of scientific studies. NLO crystals has wide application in optical modulation, optical switching, optical logic, frequency conversion, high speed information processing, optical communication and optical data storage. New technologies based on principle of optics and electronics emerge a new branch optoelectronics and high seed signal processing in communication technologies.
\end{abstract}

\section{Introduction:}

Present day advanced technologies heavily rely on one particular class of matter, i.e. the crystals. It is the periodic nature of the atoms and the properties arising due to the periodicity in crystals that is exploited to meet various technological feats. The technological revolutions in the semiconductor, optics and communication industries are the examples. The anisotropy in the crystals gives them enhanced properties as required in the field of non-linear optics. The field of non-linear optics became practically a reality after the invention of lasers. The coherent and monochromatic optical beams in the visible and ultraviolet ranges are in high demand due to their application in the fields like material processing, semiconductor lithography, laser micromachining, laser spectroscopy, photochemical synthesis, inertial confinement fusion and other basic scientific studies.

Crystals have intrigued mankind since ancient times. The main applications of crystals till the 19th century were as precious stones in jewellery, in medical prescriptions, and in mysticism because of the belief in special virtues of gemstones - a superstition still existing in modern times. Only in the 17 th-18th centuries, when the two principal laws governing crystal habits were discovered, crystallography became an independent branch of science. Earlier it was connected with mineralogy, the most perfect objects of investigation of which were crystals. Later, crystallography became associated more with chemistry, because it was apparent that the habit of crystals depends directly on their composition, and can only be explained on the basis of atomic or molecular concepts. In the 20th century, crystallography became more oriented towards physics, and an increasing number of new optical, electrical, mechanical and magnetic phenomena inherent to crystals were discovered. The discovery of X-ray diffraction by crystals, early 20th century, led to a complete change in the content and substance of crystallography, as also in the whole science of the atomic structure of matter. The quest for knowledge has made mankind grow synthetic crystals as an alternative to the limited availability of natural crystals of the requisite quality or size.

\section{Result:}

Crystal growth, which was earlier a relatively narrow field of materials science, has now been identified as a thrust area among the major areas of science and technology. This became true after the discovery of novel materials which bridge the gap between technology and human life. Crystals find applications in electronics, lasers, light valves, laser communication devices $[1,2]$, light emitting diodes, thermal imaging, pyroelectric detectors [3], X-ray spectroscopy [4, 5], inertial confinement fusion [6], etc. Large crystals are usually essential for device fabrication, and it must be possible to grow crystals of good optical quality for utilization [7]. Efforts are made to grow large crystals in short durations by fastgrowth techniques.

\section{Surface Nonlinear Optics, Spectroscopy, and} Imaging:

In this section, we will dwell upon the potential of nonlinear-optical methods for the investigation of surfaces and interfaces. The ability of second-order nonlinear-optical processes, such as SHG and SFG, to probe surfaces and interfaces is most clearly seen in the case of a centrosymmetric material. In this situation, the electric-dipole SHG and SFG response from the bulk of the material vanishes. At a surface or an interface, the bulk symmetry 
is broken, and electric-dipole second-order nonlinear-optical effects are allowed. Such surface-specific SHG and SFG processes enable a highly sensitive nondestructive local optical diagnostics of surfaces and interfaces (Figure). Illuminating and physically insightful discussion of this technique can be found in the classical texts on nonlinear optics. The invention of the laser in 1960 opened many new fields of research. One of them is nonlinear optics triggered by Franken with the first demonstration of frequency doubling in a crystal (1961) and pioneered by Bloombergen and coworkers. Third-harmonic generation in gases was observed for the first time by New and Ward in 1967, fifth and higher-order harmonic generation a few years later by Reintjes and others. The main objectives of this research were to increase the conversion efficiency, to cover a large spectral range with, for example, frequency mixing processes and to reach very short wavelengths. The most natural route for the latter goal was to use fundamental fields with the shortest possible wavelength, to produce short-wavelength radiation through a low-order nonlinear-optical process. Another research area, which started in the late sixties, is the study of atoms in strong laser fields. The objective of this fundamental research was simply to understand the behavior of atoms and molecules exposed to intense electromagnetic fields. This field evolved in parallel with the development of pulsed lasers towards increasing peak powers, increasing repetition rates and decreasing pulse durations.

\section{Conclusion:}

Indeed, efficient photon emission in the extreme ultraviolet (XUV) range, in the form of high-order harmonics of the fundamental laser field, was observed for the first time in 1987, in Saclay (33 ${ }^{\text {rd }}$ harmonic of a Nd:YAG laser) and in Chicago (17 th harmonic of a $\mathrm{KrF}$ laser).

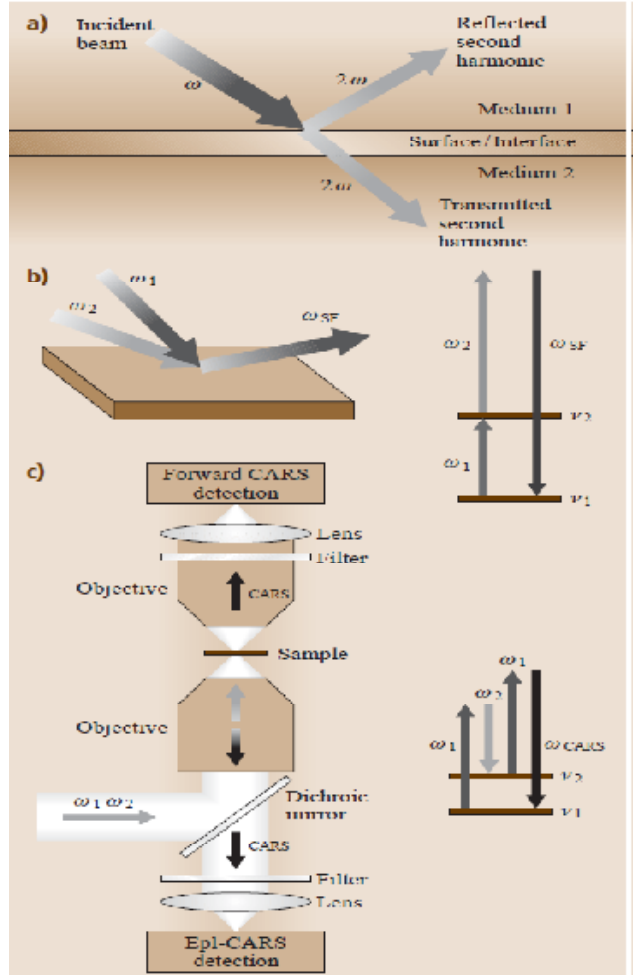

Figure. 1

\section{References :}

[1] J. Carmine Salvo, IEEE Trans. Electron Dev. 18 (1971) 748.

[2] G. Marie, J. Donjon, R. Le Pape, B. Monod, Onde Electrique 54 (1974) 421.

[3] S. Satapathy, S.K. Sharma, A.K. Karnal, V.K. Wadhawan, J. Crystal Growth 240 (2002) 196.

[4] K.B.R. Verma, A.R. Raju, K.J. Rao, Cryst. Res. Technol. 23 (1988) 185.

[5] K. Srinivasan, K. Meera, P. Ramasamy, J. Crystal Growth 205 (1999) 457.

[6] N. Zaitseva, J. Atherton, R. Rozsa, L. Carman, I. Smolsky, M. Runkel, R. Ryon, L. James, J. Crystal Growth 197 (1999) 911.

[7] C.L. Tang, Handbook of Optics, Chapter 38, Vol. II, McGraw-Hill (1995). 